\title{
Estrés académico y sintomatología de gastritis en los beneficiarios del comedor Universitario, Chachapoyas, 2017
}

\section{Academic stress and gastritis symptoms in dining room beneficiaries University, Chachapoyas, 2017}

\author{
Jeiner Jimy Tirado Cusma, ${ }^{1}$ Sonia Tejada Muñoz ${ }^{2}$
}

\begin{abstract}
RESUMEN
El presente estudio fue de enfoque cuantitativo, relacional, observacional, prospectivo, transversal y analítico cuyo objetivo fue determinar la relación que existe entre el estrés académico y la sintomatología de gastritis en beneficiarios del comedor de la Universidad Nacional Toribio Rodriguez de Mendoza de Amazonas, Chachapoyas - 2017. Se tuvo una muestra de 45 beneficiarios categorizados. Para la recolección de datos se utilizó el método de la encuesta, técnica del cuestionario, los instrumentos utilizados fueron: inventario SISCO de estrés académico con confiabilidad de 0.90 y el test de sintomatología de gastritis validado mediante la prueba binomial cuyo valor fue 8.88 (adecuado) y la determinación de confiabilidad por Alfa de Crombach obteniéndose un valor de 0.85 (alta confiabilidad). Los resultados fueron: el $71.1 \%$ presentaron sintomatología de gastritis con estrés académico moderado, el $20 \%$ no presentó sintomatología de la gastritis y tiene estrés académico leve y el $8.9 \%$ no tiene sintomatología de gastritis y tienen estrés académico moderado. La hipótesis fue contrastada con la prueba estadística no paramétrica de la Ji-cuadrada $\mathrm{X}^{2}=27.692 ; \mathrm{Gl}=2 ; \mathrm{p}=0.023<\alpha=$ 0.05 , aceptando la Hipótesis alterna $\left(\mathrm{H}_{*}\right)$. Concluyéndose que el estrés académico tiene relación significativa con la sintomatología de la gastritis.
\end{abstract}

Palabras clave: Estrés, estrés académico, síntoma, gastritis, beneficiario, comedor.

\begin{abstract}
This study was quantitative, relational, observational, prospective, transversal and analytical approach whose objective was to determine the relationship between academic stress and symptoms of gastritis in beneficiaries Eater National Toribio Rodriguez Universidad de Mendoza of Amazonas, Chachapoyas - 2017. A sample of 45 recipients were categorized I had. the method of the survey questionnaire technique was used for data collection instruments used were: inventory SISCO academic stress reliability of 0.90 and test symptoms of gastritis validated using the binomial test whose value was 8.88 (adequate) and determining reliability of Alfa Cronbach obtaining a value of 0.85 (high reliability). The results were: $71.1 \%$ had moderate symptoms of gastritis with academic stress, $20 \%$ had no symptoms of gastritis and has mild academic stress and $8.9 \%$ have no symptoms of gastritis and have moderate academic stress. The hypothesis was contrasted with the non-parametric statistical testChi-square $\mathrm{X} 2=27,692 ; \mathrm{Gl}=2 ; \mathrm{p}=0.023<\alpha=0.05$, acceptingthe alternative hypothesis (Ha). It concluded that academic stress is significantly associated with symptoms of gastritis.
\end{abstract}

Keywords: Stress, academic stress, symptom, gastritis, beneficiary, dining room.

\footnotetext{
${ }^{1}$ Licenciado en Enfermería - Facultad Ciencias de la Salud de la Universidad Nacional Toribio Rodríguez de Mendoza de Amazonas. Email:sumirtc4@hotmail.com

${ }_{2}$ Doctora en ciencias de enfermería, docente asociado a tiempo completo de la Facultad de Ciencias de la Salud de la Universidad Nacional Toribio Rodríguez de Mendoza de Amazonas.
} 


\section{Introducción}

La tercera parte de la población universitaria vive en un estado de estrés extremo, mientras que cerca de la mitad (48\%) considera que su estrés ha aumentado en los últimos cinco años (OMS, 2015, p. 28).

El $67 \%$ de la población universitaria en el Perú padece de estrés académico y es considerado un grupo sometido a una actividad homogénea constituido por períodos particularmente estresantes. Las exigencias académicas, horarios, las nuevas relaciones interpersonales, los tiempos de entrega de los trabajos prácticos hacen que, independientemente de asistir a una universidad pública o privada, los seres humanos que en ese momento juegan el rol de estudiantes, vivencien los años universitarios como estresantes (Martínez, 2015, pp. 11-12).

Es por ello que el estrés afecta cada aspecto de la vida, trabajo, relaciones personales, patrones de sueño y los hábitos de alimentación, así como a la salud, para la práctica profesional. Sin embargo, los altos niveles de estrés que reportan muchos estudiantes universitarios pueden implicar consecuencias negativas a largo plazo en la salud, que van desde fatiga, enfermedades cardiacas y la gastritis (Sanchez, 2012, p. 57).

En el Perú, ocho de cada diez estudiantes universitarios sufren gastritis y entre las principales causas se encuentra la ingesta de alimentos con exceso de grasas y condimentos, estrés académico entre otros. (Barrenechea, 2016. pp. 13-16).

La Universidad Nacional Toribio Rodríguez de Mendoza en Chachapoyas Amazonas Perú no es la excepción, toda vez que se ha evidenciado esta realidad, ya que, al aplicar un pre - test de estrés académico, a los comensales categorizados beneficiarios del comedor universitario se evidenció que: del 100\% (85) de beneficiarios categorizados, un $52.9 \%$ (45) presentó un estrés académico con sintomatología de gastritis.

En este contexto se formula el problema de investigación: ¿Cuál es la relación que existe entre el estrés académico y la sintomatología de gastritis en beneficiarios del comedor de la Universidad Nacional Toribio Rodríguez de Mendoza de Amazonas, Chachapoyas, 2017?, cuyo propósito fue determinar la relación que existe entre el estrés académico y la sintomatología de gastritis en estudiantes beneficiarios del comedor universitario.

\section{Material y métodos}

Esta investigación es de enfoque cuantitativo, observacional, prospectivo, transversal y analítico (Supo, 2015,pp. 2 - 18).

La muestra estuvo conformada por 45 beneficiarios categorizados del comedor universitario de esta universidad, seleccionados de acuerdo a los criterios de inclusión por muestreo no probabilístico por conveniencia

Métodos, técnicas e instrumentos de recolección de datos: Para ambas variables se aplicó el método de la encuesta y la técnica del cuestionario (Canales, 1994, p. 275).

El instrumento de recolección de datos para la variable estrés académico fue el Inventario SISCO del estrés académico, consiste en 15 ítems el cual estuvo dividido en tres dimensiones: física, psicológica y comportamental. Dicho instrumento muestra una confiabilidad de 0.90 y una validez de 0.80 . $\mathrm{Su}$ estructura consiste en 15 ítems, en un escalamiento tipo likert. Las categorías utilizadas fueron: Estrés académico leve, moderado y severo (Barranza, 2007, p. 12). Para la variable de Sintomatología de la gastritis, se utilizó el "test de sintomatología de la gastritis", validado mediante prueba binomial y obteniendo un valor de 8.88 (adecuado). La confiabilidad se determinó por Alfa de Crombach cuyo valor obtenido fue 0.85 (alta confiabilidad). El test consistente en 13 ítems, presentado en forma de preguntas de dos opciones en un formato de la escala dicotómica. Las categorías utilizadas fueron: con sintomatología y sin sintomatología.

Para contrastar la hipótesis se utilizó la prueba estadística no paramétrica del ji - cuadrado con nivel de significancia del $\alpha=0.05$ (95\% de confiabilidad y $5 \%$ del margen de error).

\section{Resultados}

Tabla 01: Estrés académico en los beneficiarios del comedor universitario

\begin{tabular}{lcc}
\hline Estrés académico & & \\
\hline & $\mathbf{f i}$ & $\mathbf{\%}$ \\
\hline Leve & 9 & 20 \\
Moderado & 36 & 80 \\
Severo & 00 & 00 \\
\hline TOTAL & $\mathbf{4 5}$ & $\mathbf{1 0 0}$ \\
\hline
\end{tabular}

Fuente: Inventario SISCO de estrés académico. 
En la tabla 01, se observa que del 100\% (45) de estudiantes beneficiarios categorizados del comedor de la UNTRM, el 80\% (36) tienen un tipo de estrés académico moderado, el 20\% (9) leve y $0 \%$ severo.

Tabla 02: Sintomatológica de gastritis en los beneficiarios del comedor universitario

\begin{tabular}{lcc}
\hline \multicolumn{2}{l}{ Sintomatología de la gastritis } & \\
\hline fi & $\%$ \\
Con sintomatología & 32 & 71.1 \\
Sin sintomatología & 13 & 28.9 \\
\hline TOTAL & $\mathbf{4 5}$ & $\mathbf{1 0 0}$ \\
\hline
\end{tabular}

En la tabla 02, se observa que del 100\% (45) estudiantes beneficiarios categorizados del comedor de esta universidad, el $71.1 \%$ (32) tienen sintomatología de la gastritis y el $28.9 \%$ (13) no presentan.

Tabla 03: Relación entre el estrés académico y sintomatológica de gastritis en los beneficiarios del comedor universitario.

\begin{tabular}{|c|c|c|c|c|c|c|c|c|}
\hline \multirow{3}{*}{ Sint.gast. } & \multicolumn{6}{|c|}{ Estrés académico } & \multirow{2}{*}{\multicolumn{2}{|c|}{ TOTAL }} \\
\hline & \multicolumn{2}{|c|}{ Leve } & \multicolumn{2}{|c|}{ Mod. } & \multicolumn{2}{|c|}{ Sev. } & & \\
\hline & fi & $\%$ & fi & $\%$ & fi & $\%$ & fi & $\%$ \\
\hline $\begin{array}{l}\text { Con } \\
\text { Sint. }\end{array}$ & 0 & 0 & 32 & 71.1 & 0 & 0 & 32 & 71.1 \\
\hline Sin Sint. & 9 & 20 & 4 & 8.9 & 0 & 0 & 13 & 28.9 \\
\hline TOTAL & 9 & 20 & 36 & 80 & $\mathbf{0}$ & $\mathbf{0}$ & 45 & 100 \\
\hline
\end{tabular}

$\mathrm{X}^{2}=27.692 ; \mathrm{Gl}=2 ; \mathrm{p}=0.023<\alpha=0.05 \quad(*)$

$(*) \quad$ : Significativo

En la tabla 03 , se observa que del $100 \%$ de estudiantes beneficiarios categorizados del comedor universitario, el 71.1\% (32) tiene sintomatología de gastritis con un estrés académico moderado. Así mismo el 20\% (9) no presenta sintomatología de la gastritis pero tiene un estrés académico leve y el $8.9 \%$ (4) no tiene sintomatología de gastritis pero tienen un estrés académico moderado.

\section{Discusión}

Tolentino (2009), afirmó que los estudiantes universitarios del tercer semestre presentaron un nivel de estrés medio en un $30 \%$, mientras que los del cuarto semestre en un 26\%. Asimismo Bedoya, (2006) señala que los estudiantes del tercer y cuarto año de la Facultad de Estomatología de la Universidad Peruana Cayetano Heredia, Lima Perú en un $96.6 \%$ presentaron un nivel de estrés académico alto. Boullosa, (2013) concluyó que el 44\% de estudiantes universitarios presentó un estrés académico moderado, el 52\% leve y el $4 \%$ niveles de estrés severo.

El presente estudio evidencia un nivel de estrés moderado, evidenciándose similitud con el estudio realizado por Tolentino (2009) quien obtuvo un estrés de nivel medio en su mayoría, teniendo diferencias con las investigaciones realizadas por Bedoya (2006) y Boullosa (2013) quienes obtuvieron nivel de estrés alto y leve respectivamente.

Al respecto la vida universitaria puede generar estrés, por organizar horarios para trabajar y estudiar y hasta garantizar que lo que ha elegido responde a una vocación. Sin embargo, las carreras profesionales de la salud presentan mayor estrés académico. Los estudiantes de la Facultad Ciencias de la Salud, sufren un importante estrés desde el comienzo de su formación académica y si bien es aceptable cierto grado de tensión, no todos los estudiantes lo resuelven de manera adecuada. En muchos de ellos, los programas y las exigencias generan miedo, incompetencia, enojo y sensación de inutilidad y culpa. Todas estas manifestaciones pueden producir respuestas psicológicas y físicas; causando problemas de salud, como enfermedades crónicas, cardiacas, gastrointestinales. (OMS, 2015, p. 21).

En contraste con Carrasco (2016), en su investigación afirmó que la frecuencia más alta de gastritis en universitarios es de $55.3 \%$, mientras que el $44.7 \%$ no presentan. La mayoría de estudiantes universitarios presentaron síntomas gástricos debido al estrés académico prolongado al que estuvieron expuestos. Sin embargo hay similitud con la presente investigación y el estudio de Herrera, (2012), donde señaló que la prevalencia de gastritis en estudiantes universitarios es de $31 \%$, y el $6 \%$ no presentan.

Por consiguiente, el estudio de Carrasco (2016) y Herrera (2012) tienen similitud con la presente investigación ya que en ambos resultados los estudiantes presentaron porcentajes elevados de sintomatología de gastritis.

Los estudiantes universitarios por su rutina de estudio no tienen horas fijas de alimentación y están expuestos a desarrollar gastritis, además la vida de los fines de semana con el consumo de alcohol, fiestas y el excesivo consumo de alimentos dañinos son las principales causas de esta situación, actualmente los 
jóvenes ingieren bebidas alcohólicas desde temprana edad, además del tabaco, gaseosa, comida chatarra y picante, que aumentan la acidez en el estómago de la persona (Valenzuela, 2004).

Las alteraciones de los neurotransmisores implicados en el estrés, como son la acetilcolina o la histamina, podrían modificar diferentes funciones orgánicas a través de sus efectos sobre los sistemas nerviosos central y periférico y producir un aumento de la secreción de ácido gástrico, así como la reducción de la barrera moco defensiva de la mucosa gástrica causante de la acidez. Este vínculo entre el estrés y la acidez estomacal se debe a la estrecha interacción entre el sistema nervioso y el digestivo. (Organización Peruana de la Salud 2011, pp. 32 43).

Finalmente los resultados de la prueba estadística no paramétrica $\mathrm{Ji}$ - cuadrado $\left(\mathrm{X}^{2}=27.692 ; \mathrm{Gl}=1 ; \mathrm{p}=\right.$ $0.023>\alpha=0.05)$ aplicada a esta tabla de contingencia indican que existe una relación directa estadísticamente significativa entre el estrés académico y la sintomatología de la gastritis.

Los resultados de la presente investigación difieren con la investigación realizada por Borbor (2016), donde concluyó que el $92 \%$ de estudiantes universitarios manifiesta síntomas de gastritis y estrés académico, mientras que en un $8 \%$ no lo presentó.

Es pertinente afirmar que los estudiantes beneficiarios del Comedor universitario presentan estrés y sintomatología de gastritis lo que implica proponer esfuerzos compartidos para realizar estudios de diagnóstico más sofisticados como endoscopías y biopsias y determinar el agente causal de la gastritis y poder brindar tratamiento oportuno y de esta manera contribuir a reducir los índices de cáncer por $H$. pilori, en esta población de estudio; procesos que constituyeron una limitante en esta investigación ya que implica contar con recursos materiales y humanos sofisticados que demandan altos costos para los investigadores.

\section{Conclusiones}

- Los estudiantes universitarios beneficiarios del comedor, presentaron predominantemente porcentajes más elevados de estrés académico moderado en un $80 \%$.

- Los estudiantes universitarios beneficiarios del comedor presentaron sintomatología de gastritis en un $71.1 \%$.

- Existe diferencia significativa entre el nivel de estrés y la sintomatología de gastritis en beneficiarios del comedor Universitario de Chachapoyas, 2017.

\section{Agradecimiento}

- A los estudiantes universitarios beneficiarios del comedor de la Universidad Nacional Toribio Rodríguez de Mendoza de Amazonas, por permitirnos entrar en sus vidas privadas durante el proceso de recolección de datos, el mismo que sirvió para la respectiva tabulación, procesamiento y análisis de los resultados.

- A la Directora de la Dirección de Bienestar Universitario y Deporte, de la Universidad Nacional Toribio Rodriguez de Mendoza de Amazonas por brindarnos las facilidades del caso para el proceso de recolección de datos.

\section{Referencias bibliográficas}

Barranza, A. (2013). El estrés académico. Durango: Adneture work.

Barrenechea, O. (2016). Índice de gastritis en el Perú. Lima: FASA.

Bedoya, S (2006). Evaluación de niveles, situaciones generadoras y manifestaciones de estrés académico en alumnos de tercer y cuarto año de la facultad de estomatología durante el semestre 2005- I. (Tesis para optar el título de cirujano dentista). Lima.

Boullosa, G. (2013). Estrés académico y afrontamiento en un grupo de estudiantes de la Pontificia Universidad Católica del Perú. (Tesis para optar el título de Licenciada en Psicología, con mención en Psicología Clínica). Lima.

Borbor, K. (2016). Estrés académico como factor causal de gastritis en estudiantes universitarios UPSE - 2016. (Tesis para optar el título de Licenciada en Enfermería). La Libertad.

Canales. (1994). Metodología de la investigación. Buenos Aires: Worksst.Carrasco, G. (2016). Incidencia y prevalecía de gastritis en estudiantes universitarios de la Escuela Superior Politécnica de Chimborazo. (Tesis para optar el título de licenciatura de Medicina). Ecuador

Herrera, E. (2012). Prevalencia de gastritis en un segmento de la población estudiantil de la Facultad de Ciencias Químicas y Farmacia de la Universidad de San Carlos de Guatemala que cursan el segundo año con carnet 2010. (Tesis de Licenciatura en Química Farmacéutica). 
Universidad de San Carlos de Guatemala. Guatemala.

Martinez, E. (2015). Una aproximación psicosocial al estrés universitario. Madrid.

OMS. (2015). Gastritis en universitarios. California: Granpryt.Sanchez, J. (2012). 101 secretos para una vida sana. Buenos Aires: Casa editora sudamericana.

Supo, J. (2015). Investigación cientifica I. Jalisco: Adventure.Tolentino, S. (2009). Perfil de estrés académico en alumnos de licenciatura en psicología. (Tesis de Licenciatura en Psicología). Universidad Autónoma de Hidalgo. Actopan.

Valenzuela, J. (2004). Diagnóstico y Tratamiento de las Enfermedades Digestivas. Santiago de Chile: IKU. 